\title{
Blue Organic LEDs With Improved Power Efficiency
}

\author{
H. M. Zhang, Wallace C. H. Choy, Senior Member, IEEE, and K. Li
}

\begin{abstract}
High-power-efficiency blue fluorescent organic lightemitting devices have been demonstrated by simultaneously doping two hole-conduction layers of 4, 4', 4"-Tris(N-3methylphenyl-N-phenyl-amino) triphenylamine (m-MTDATA), and $\mathrm{N}, \mathrm{N}^{\prime}$-diphenyl-N, $\mathrm{N}^{\prime}$-bis(1-naphthyl)-(1, 1'-biphenyl)-4, $4^{\prime}$-diamine (NPB) with 2,3,5,6-tetrafluoro-7,7,8,8-tetracyanoquinodimethane (F4-TCNQ) as well as doping two ambipolar emission layers with $p$-bis( $p$-N, N-diphenyl-aminostyryl) benzene (DSA-Ph). By doping the two host layers with DSA-Ph to form the double emission layer, the current efficiency is enhanced due to the extended emission zone. We further increase the performance by introducing the doping F4-TCNQ into the hole-injection and transporting layers to reduce the transport barrier at the m-MTDATA:F4TCNQ/NPB and to enhance the hole injection and conduction. The luminance and power efficiencies reach $8.9 \mathrm{~cd} / \mathrm{A}$ and $4.5 \mathrm{~lm} / \mathrm{W}$, respectively.
\end{abstract}

Index Terms-Blue light emission, doping hole injection layer, double emission layer, organic LEDs, power efficiency.

$\mathbf{H}$ IGHLY efficient blue light organic light-emitting devices (OLEDs) have attracted great interests from both academia and industries for solid-state lighting and full-color display applications. For the blue electrophosphorescent OLEDs where both singlet and triplet excitons contribute to the light emission, there is an advantage, over the conventional blue electrofluorescent OLEDs, of theoretical 100\% internal quantum efficiency. However, there are limited appropriate host materials which have a higher triplet-excited-state energy than that of phosphorescent dopants for realizing exothermic energy transfer [1]. There is also exchange energy loss existing in the process of intersystem crossing from singlet to triplet excited state. Moreover, blue electrophosphorescent OLEDs generally have short lifetimes and large roll-off issues as compared to their fluorescent counterparts [2]. Hence, blue electrofluorescent OLEDs are still attractive, and various approaches have been proposed to improve the efficiency and color emission quality [2]-[5]. The guest-host system through doping fluorescent dye into the emitting layer (as host) has been used extensively for improving the performance.

In addition, the confinement of excitons in the recombination zone is critical for enhancing the device efficiency for avoiding the losses of radiative excitons [6]. In order to utilize the generated excitons as much as possible for light emission, it

Manuscript received May 11, 2009; revised September 22, 2009. First published November 17, 2009; current version published December 23, 2009. This work was supported by the Research Grants Council of the HK Special Administrative Region, China, under Grant HKU712108. The review of this paper was arranged by Editor M. J. Kumar.

The authors are with the Department of Electrical and Electronic Engineering, The University of Hong Kong, Hong Kong (e-mail: chchoy@eee.hku.hk).

Digital Object Identifier 10.1109/TED.2009.2033641 is necessary to increase the width of the recombination zone for high efficiency such as by using double emission layers (DELs) [7]-[9]. Meanwhile, carrier injection and transport also play an important role for the performance of OLEDs. The hole injection can be improved by introducing dopants such as 2,3,5,6-tetrafluoro-7,7,8,8-tetracyano-quinodimethane (F4-TCNQ) into the hole-injection layer (HIL). However, there are limited studies in simultaneously introducing the doped HIL and doped hole transport layer (HTL) as well as the fluorescent-dye-doped DEL for enhancing the performance of blue OLEDs including the $I-V$ characteristics, current, and power efficiencies.

In this paper, we report high-efficiency blue fluorescent OLEDs based on DEL using $p$-bis $(p-\mathrm{N}, \mathrm{N}$-diphenylaminostyryl) benzene (DSA-Ph) as emitter. Blue OLEDs with DEL show remarkably improved efficiency; a peak luminance efficiency $\left(\eta_{\max }\right)$ of $9.3 \mathrm{~cd} / \mathrm{A}$, a peak power efficiency of $2.5 \mathrm{~lm} / \mathrm{W}$, and a maximum luminance of more than $45000 \mathrm{~cd} / \mathrm{m}^{2}$ have been achieved. We then further improve the efficiencies by doping both HIL and HTL by F4-TCNQ. The power efficiency is improved to $4.5 \mathrm{~lm} / \mathrm{W}$. This power efficiency enhancement mainly results from the improvement of hole injection and transport.

The blue OLEDs were fabricated by vacuum thermal evaporation onto indium-tin-oxide (ITO)-coated glass substrates with a sheet resistance of $20 \Omega / \square$. Prior to organic layer growth, ITO substrates were cleaned with solvents and then exposed to UVozone ambient for $15 \mathrm{~min}$. All organic materials were grown at the base pressure of $\sim 10^{-6}$ torr with the evaporation rate around $1.0 \AA / \mathrm{s}$. The organic materials include a $4,4^{\prime}, 4^{\prime \prime}$-Tris $(\mathrm{N}-3$ methylphenyl-N-phenyl-amino) triphenylamine (m-MTDATA) doped with 4-wt\% F4-TCNQ as HIL, N, N'-diphenyl-N, $\mathrm{N}^{\prime}$-bis(1-naphthyl)-(1, 1'-biphenyl)-4, 4'-diamine (NPB) doped with 3 wt \% and without F4-TCNQ as HTL, an NPB doped with 3-wt\% DSA-Ph and a 2-methyl-9, 10-di(2-napthyl)anthracene (MADN) doped with 3-wt\% DSA-Ph as the blue emission layer system in DEL OLEDs or only MADN doped with 3-wt\% DSA-Ph as the blue single emission layer of OLEDs, and a tris(8-hydroxyquinoline) aluminum $\left(\mathrm{Alq}_{3}\right)$ as the electron transport layer. Finally, a $\mathrm{LiF}(1 \mathrm{~nm}) / \mathrm{Al}(100 \mathrm{~nm})$ cathode was deposited and patterned using a shadow mask to define an active device area of $3.57 \mathrm{~cm}^{2}$. The six prepared devices have structures as shown in Table I, and the devices have the same cathode of $\mathrm{LiF}(1 \mathrm{~nm}) / \mathrm{Al}(70 \mathrm{~nm})$.

The current-density-voltage-luminance $(J-V-L)$ characteristics of the blue OLEDs were obtained using a Keithley 2400 source and a calibrated Si photodiode. The electroluminescence (EL) spectra were recorded by an Oriel spectrometer with Cornerstone 260i. All measurements were carried out under ambient atmosphere. 
TABLE I

A-F Device Structures. They Have the Same CATHODE OF LiF $(1 \mathrm{~nm}) / \mathrm{Al}(70 \mathrm{~nm})$

\begin{tabular}{|l|l|l|l|l|l|l|}
\hline & \multicolumn{5}{|c|}{ Thickness (nm) } \\
\hline Device & $\begin{array}{l}\text { m-MTDATA: } \\
\text { F4TCNQ }\end{array}$ & $\begin{array}{l}\text { NPB: } \\
\text { F4TCNQ }\end{array}$ & NPB & $\begin{array}{l}\text { NPB: } \\
\text { DSA-ph }\end{array}$ & $\begin{array}{l}\text { MADN: } \\
\text { DSA-ph }\end{array}$ & Alq3 \\
\hline A & 20 & - & 120 & - & 40 & 18 \\
\hline B & 20 & - & 90 & - & 40 & 18 \\
\hline C & 20 & - & 90 & 20 & 40 & 20 \\
\hline D & 20 & - & 60 & 26 & 40 & 20 \\
\hline E & 20 & 9 & 75 & & 40 & 20 \\
\hline F & 20 & 9 & 60 & 26 & 40 & 20 \\
\hline
\end{tabular}

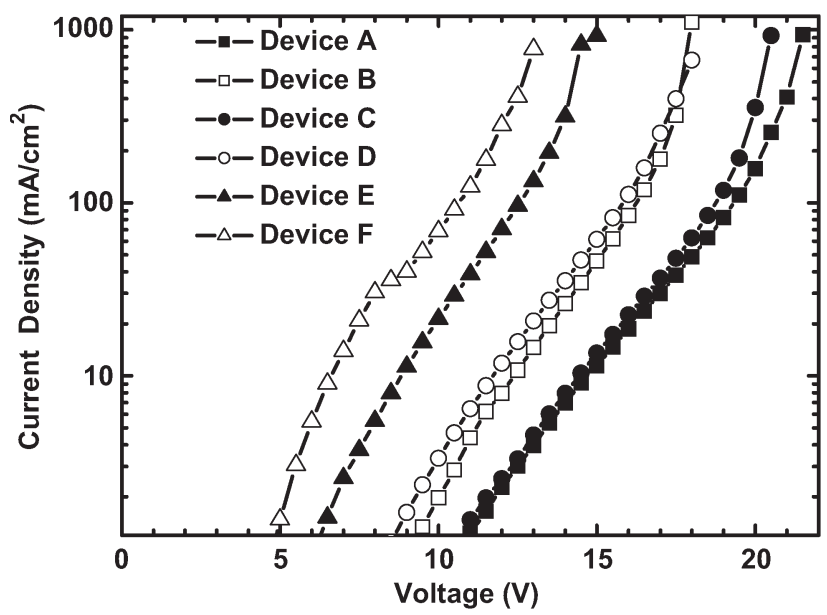

Fig. 1. $J-V$ plot of Devices A-F.

The $J-V$ characteristics of Devices A-F are shown in Fig. 1. Device A with a thicker NPB of $120 \mathrm{~nm}$ shows lower current density than Device B with the 90-nm-thick NPB at the same biased voltage due to the relatively poor hole conduction. Similarly, Device C with a 90-nm-thick NPB also shows lower current density than Device D with a 60-nm-thick NPB, when both Devices $\mathrm{C}$ and $\mathrm{D}$ are constructed from the DEL structure, even though the thickness of the emission layer of NPB:DSA-ph of Device D is slightly thicker than that of Device C. The results indicate that the thickness of the NPB has great impact on the hole conduction and, thus, the operation voltage and other performances of the OLEDs such as the luminance and power efficiencies, as will be discussed later. It can also be seen from Fig. 1 that the turn-on voltage of Device $\mathrm{D}$ is very close to that of Device B. For Device D, the total thickness of NPB $(60 \mathrm{~nm})$ and NPB:DSA-ph $(26 \mathrm{~nm})$ is almost the same as that of Device B. In other words, the doping of DSA-ph into NPB for emission purposes does not cause any significant degradation to the $J-V$ characteristics of OLEDs while the thickness increase of NPB and NPB:DSH-ph layers will degrade the $J-V$ characteristics.

Meanwhile, from Fig. 1, Devices A-D with doped HIL only, i.e., only m-MTDATA doped with F4TCNQ, were are found to generally have higher turn-on voltage as compared to Devices $\mathrm{E}$ and $\mathrm{F}$. The $J-V$ characteristics of Device F with doped HIL, i.e., m-MTDATA:F4TCNQ /NPB:F4TCNQ, can be dramatically improved as compared with Device D. The turn-on voltage of Device $\mathrm{F}$ is $\sim 3 \mathrm{~V}$ lower than that of Device D. Further evidence can be found by comparing Device E with Device D. The current density of Device E is better than that of Device D
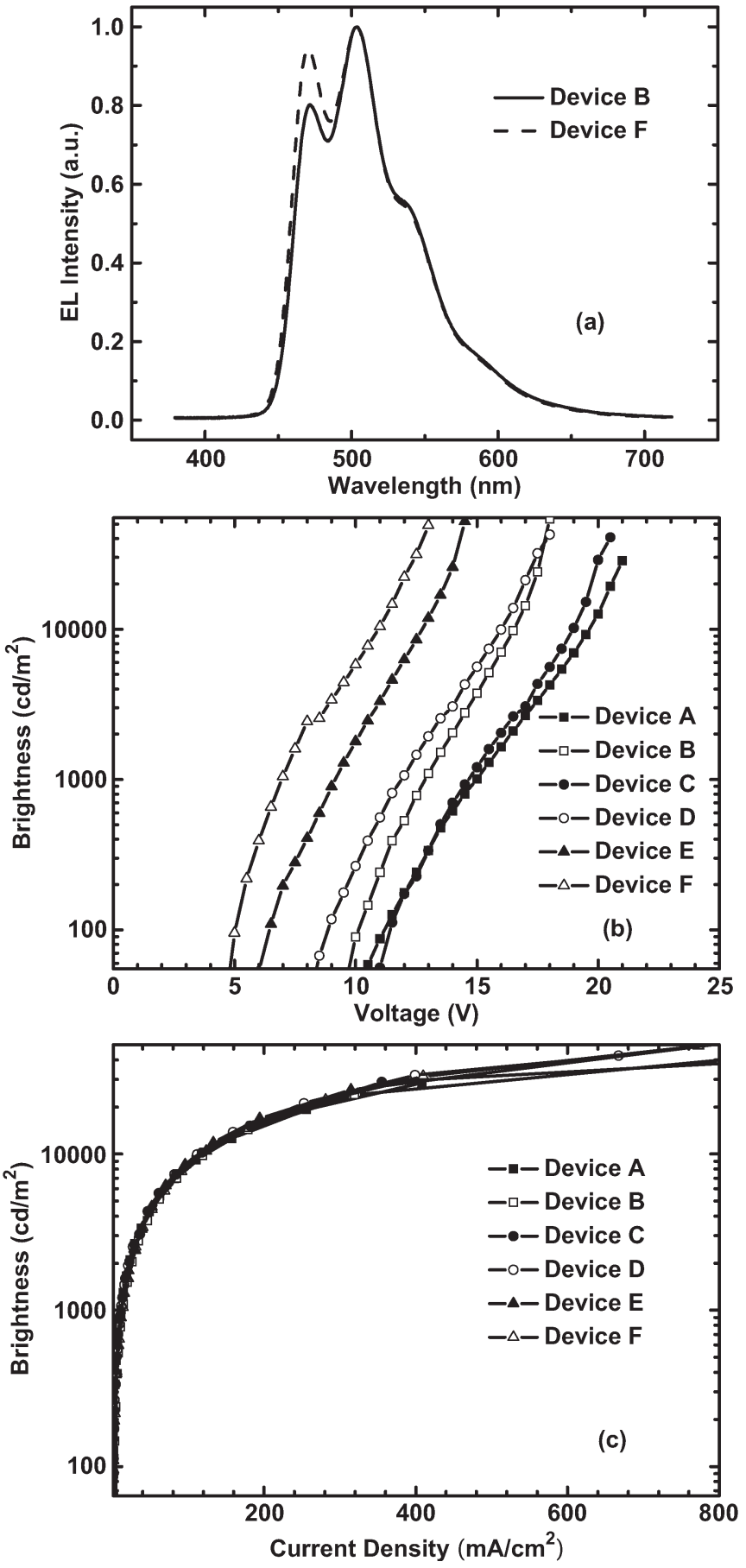

Fig. 2. (a) EL spectra of Devices B and F. (b) Brightness versus voltage and (c) brightness versus current density of Devices A-F.

due to, again, the doping of F4TCNQ into NPB. It should be noted that, as described previously, the doping of DSA-ph onto NPB will not change the hole conduction in Device D. These results are consistent with the results reported in [10].

The EL spectra of the devices are similar to each other. Here, we show the EL spectrum of Devices B and F in Fig. 2(a). The emission is mainly from the fluorescence dopant of DSA-ph. The brightness values of Devices A-F are shown in Fig. 2(b) and (c). The trends of the brightness versus voltage of the OLEDs are very similar to the $J-V$ characteristic plot. It is interesting to note that, at the same applied voltage, the brightness 

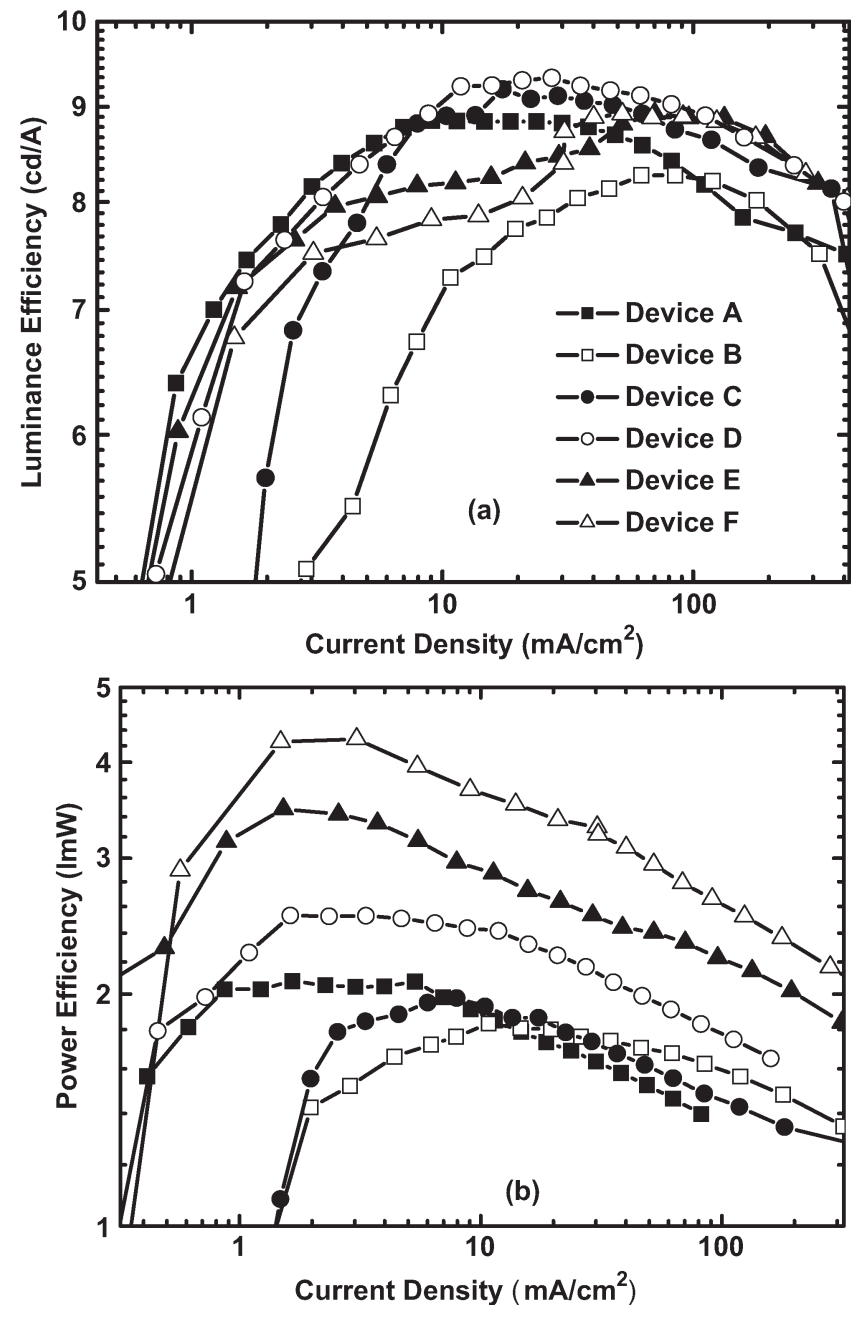

Fig. 3. (a) Luminance efficiency and (b) power efficiency of Devices A-F.

TABLE II

CURRent ANd Power EFFiciencies of Devices A-F

\begin{tabular}{cccc}
\hline Devices & $\begin{array}{c}\text { Maximum CE } \\
\text { and the current density } \\
\end{array}$ & $\begin{array}{c}\text { Maximum PE } \\
\left.\text { acd } / \mathrm{A}, \mathrm{mA} / \mathrm{cm}^{*}\right]\end{array}$ & $\begin{array}{c}\text { Turn on } \\
\text { voltage(V) }\end{array}$ \\
& $8.8,9.0$ & $2.07,5.3$ & at $1 \mathrm{~cd} / \mathrm{m}^{2}$ \\
\hline A & $8.3,62$ & $1.83,10.8$ & 9.5 \\
B & $9.2,17$ & $1.98,7.9$ & 9 \\
C & $9.3,21$ & $2.53,1.6$ & 10.5 \\
D & $8.9,70$ & $3.47,1.51$ & 7 \\
E & $8.9,52$ & $4.3,3.05$ & 5 \\
F & & & 4 \\
\hline
\end{tabular}

${ }^{*} \mathrm{CE}=$ current efficiency, $\mathrm{PE}=$ power efficiency.

of Device $\mathrm{F}$ with a thin NPB:F4TCNQ ( $9 \mathrm{~nm}$ ) layer is much larger than that of Device D without the thin layer at the same operation voltage due to the improvement of hole conduction.

The luminance and power efficiencies versus the current density of the devices are shown in Fig. 3(a) and (b), respectively. The summary is listed in Table II. From Fig. 3(a), the current efficiency of Device A is better than that of Device B, while NPB is thicker. However, when the driving current increases, the luminance efficiency of Device A drops faster, and the value is even lower than that of Device B. In addition, the trend of the power efficiency of the two devices is reverse as compared that of the current efficiency (i.e., Device A is lower than Device B) because of the higher operation voltage for Device A. As a result, the increase of NPB thickness may not be good for practical applications.

As shown in Fig. 3(a), the maximum luminance efficiency of Device D $(9.3 \mathrm{~cd} / \mathrm{A})$ with DEL and doped HIL is slightly higher than that of Device F $(8.9 \mathrm{~cd} / \mathrm{A})$ with DEL and doped HIL and HTL. However, as shown in Fig. 3(b), the maximum power efficiency of Device D is only $2.5 \mathrm{~lm} / \mathrm{W}$, which is much lower than that of Device F $(4.3 \mathrm{~lm} / \mathrm{W})$. Similarly, Device E with single doped emission layer and doped HIL and HTL has better power efficiency than the devices with doped HIL only. The result should be attributed to the doping of thin HTL (i.e., NPB:F4-TCNQ) which effectively reduces the transport barrier at the m-MTDATA:F4TCNQ/NPB interlayer and, thus, to the increase of the hole injection into HTL [10]. Therefore, the number of excitons increases at the interface of NPB:DSA-ph/ ADN:DSA-ph. Consequently, the simultaneous introduction of doped HIL and HTL is important for improving the power efficiency of OLEDs.

Furthermore, by comparing Devices F and E, where both devices have simultaneously doped HIL and HTL, it can be observed that Device F with DEL has higher power efficiency than Device E with single doped emission layer at all driving currents. We can therefore conclude that the introduction of both the DEL and the simultaneously doped HIL and HTL can improve the power efficiency but the increase of NPB thickness will degrade the power efficiency.

In summary, we show that, by using p-dopant F4-TCNQ into the hole-injection and transport layers and by simultaneously doping two ambipolar hosts MADN and NPB with the fluorescent emitter of DSA-Ph, the power efficiency of OLEDs can be significantly enhanced. Our results also show that the doping of DSA-ph into NPB for emission purposes does not cause any significant degradation to the $J-V$ characteristics of OLEDs. It should be noted that, since the thickness increase of NPB and NPB:DSA-ph layers will degrade the $J-V$ characteristics, appropriate layer thicknesses of 60 and $26 \mathrm{~nm}$, respectively, have been used for achieving the luminance and power efficiencies of $8.9 \mathrm{~cd} / \mathrm{A}$ and $4.5 \mathrm{~lm} / \mathrm{W}$, respectively.

\section{REFERENCES}

[1] C. Adachi, R. C. Kwong, P. Djurovich, V. Adamovich, M. A. Baldo, M. E. Thompson, and S. R. Forrest, "Endothermic energy transfer: A mechanism for generating very efficient high-energy phosphorescent emission in organic materials," Appl. Phys. Lett., vol. 79, no. 13, pp. 2082-2084, Sep. 2001.

[2] S. W. Wen, M. T. Lee, and C. H. Chen, "Recent development of blue fluorescent OLED materials and devices," J. Display Technol., vol. 1, no. 1, pp. 90-99, 2005

[3] Z. Q. Gao, B. X. Mi, C. H. Chen, K. W. Cheah, Y. K. Cheng, and W. S. Wen, "High-efficiency deep blue host for organic light-emitting devices," Appl. Phys. Lett., vol. 90, no. 12, pp. 123 506-1-123 506-3, Mar. 2007.

[4] M. T. Lee, H. H. Chen, C. H. Liao, C. H. Tsai, and C. H. Chen, "Stable styrylamine-doped blue organic electroluminescent device based on 
2-methyl-9, 10-di(2-naphthyl)anthracene," Appl. Phys. Lett., vol. 85, no. 15, pp. 3301-3303, Oct. 2004.

[5] Y. Sun, N. C. Giebink, H. Kanno, B. Ma, M. E. Thompson, and S. R. Forrest, "Management of singlet and triplet excitons for efficient white organic light-emitting devices," Nature, vol. 440, no. 7086, pp. 908912, Apr. 2006.

[6] B. D. Chin and C. Lee, "Confinement of charge carriers and excitons in electrophosphorescent devices: Mechanism of light emission and degradation," Adv. Mater., vol. 19, no. 16, pp. 2061-2066, Aug. 2007.

[7] G. He, M. Pfeiffer, K. Leo, M. Hofmann, J. Birnstock, R. Pudzich, and J. Salbeck, "High-efficiency and low-voltage p-i-n electrophosphorescent organic light-emitting diodes with double-emission layers," Appl. Phys. Lett., vol. 85, no. 17, pp. 3911-3913, Oct. 2004.

[8] J. W. Kang, S. H. Lee, H. D. Park, W. I. Jeong, K. M. Yoo, Y. S. Park, and J. J. Kim, "Low roll-off of efficiency at high current density in phosphorescent organic light emitting diodes," Appl. Phys. Lett., vol. 90, no. 22, pp. 223 508-1-223 508-3, May 2007.

[9] T. Zheng and W. C. H. Choy, "High-efficiency blue fluorescent organic light emitting devices based on double emission layers," J. Phys. D, Appl. Phys., vol. 41, no. 5, pp. 055 103-1-055 103-5, Mar. 2008.

[10] S. W. Tsang, Z. H. Lua, and Y. Tao, "Engineering carrier transport across organic heterojunctions by interface doping," Appl. Phys. Lett., vol. 90, no. 13, pp. 132 115-1-132 115-3, Mar. 2007.

H. M. Zhang, photograph and biography not available at the time of publication.
Wallace C. H. Choy (SM'07) received the Ph.D. degree in electronic engineering from the University of Surrey, Guildford, U.K.

His work at Surrey was supported by the Croucher Foundation Scholarship. He then joined the National Research Council (NRC) of Canada as a member of the research staff to work on optical device structures of polarizationindependent optical amplifiers and modulators. In 2001, he joined Fujitsu, San Jose, CA, to develop real-time wavelength tunable lasers and optical transmitter modules. He is currently an Assistant Professor with the Department of Electrical and Electronic Engineering, University of Hong Kong (HKU), Pokfulam, Hong Kong. He has published more than 75 internationally technical journal papers and contributed to two book chapters. He is the holder of one U.S. patent. His current research interests include organic optoelectronic devices and nanomaterial devices.

Dr. Choy was the recipient of the Outstanding Achievement Award from the National Research Council of Canada in 2001; the Sir Edward Youde Memorial Fellowship and Croucher Foundation Fellowship; and the Overseas Visiting Fellowship from HKU to take a sabbatical with G. Malliaras's Group at Cornell University, Ithaca, NY, in 2008 and to visit Prof. Y. Yang of the University of California, Los Angeles, in the summer of 2009 and to conduct research in polymer solar cells and LEDs. Since 2005, he has been a Technical Consultant for HK-Ulvac (a member of the stock-listed Ulvac Corporation). $\mathrm{He}$ has delivered invited talks and served as a committee member in international industrial and academic conferences organized by various organizations, such as the IEEE, Optical Society of America, and Plastic Electronics Foundation.

K. Li, photograph and biography not available at the time of publication. 